Antonini, A. 319

Berg, D. 291

Braune, S. 341

Del Tredici, K. 341

Esterhammer, R. 300

Gaenslen, A. 291

Hotter, A. 300

Hussl, A. 300
Isaias, I.U. 319

Jost, W.H. 283, 341

Landvogt, C. 341

Mahlknecht, P. 300

Reichmann, H. 284

Schocke, M. 300

Seppi, K. 300

Tatsch, K. 330

\title{
Subject Index Vol. 7, No. 5, 2010
}

${ }^{123}$ I-metaiodobenzylguanidine scintigraphy 341

Amyloid imaging 330

Atypical parkinsonian disorders 300

- _ syndromes 291, 341

Cardiac sympathetic denervation 330

Corticobasal degeneration 300

Diagnostic tests 284

Differential diagnosis 319, 330

Diffusion 300

Dopaminergic system 330

Essential tremor 319

Glucose metabolism 330
Hyposmia 284

Inflammation 330

Magnetic resonance imaging 300

Multiple system atrophy 300

Parkinson's disease 284, 291, 300, 319, 330,341

Positron emission tomography 330

Progressive supranuclear palsy 300

REM sleep behavior disorder 284

Single-photon emission computed tomography 319

Substantia nigra hyperechogenicity 291

Transcranial B-mode sonography 291 